\title{
Open repair of extent-III thoracoabdominal aortic aneurysm using a modified branch-first technique
}

Niranjan Hiremath, MCh, FVES, FACS(Aus), ${ }^{\mathrm{a}, \mathrm{b}}$ Houssam Younes, MD, ${ }^{\mathrm{c}}$ Tareq Aleinati, MD, ${ }^{\mathrm{b}}$ and Woosup Michael Park, MD, ${ }^{c}$ Cleveland, Ohio, and Abu Dhabi, United Arab Emirates

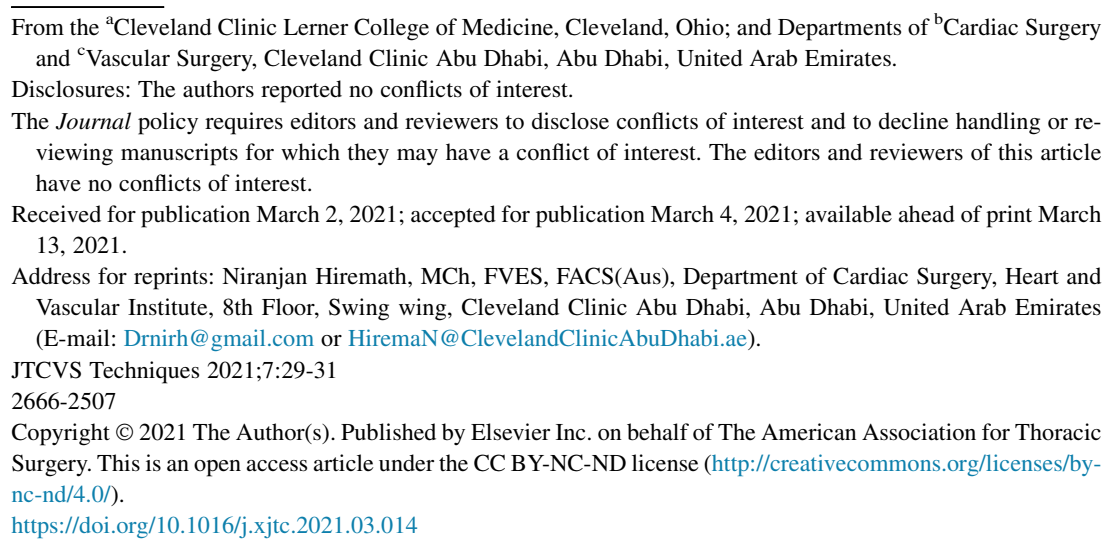

Video clip is available online.

Thoracoabdominal aortic aneurysm (TAAA) surgery is an extensive surgical undertaking, and adjuncts to decrease complications have varying results and individual pitfalls. We describe a modified "branch-first" TAAA open repair technique that avoids cardiopulmonary bypass (CPB) and still decreases the visceral ischemia time, bleeding, and other associated complications.

\section{CASE REPORT}

A 51-year-old man with history of hypertension, tobacco abuse, and chronic type B aortic dissection for which he had received medical treatment for 7 years presented with epigastric pain that radiated to the back. Computed tomography aortography revealed a $6.5-\mathrm{cm}$, extent-III TAAA with a tortuous course (Figure 1, A). He was deemed anatomically unsuitable for endovascular repair. After a multidisciplinary team discussion, the decision was made to proceed with a modified branch-first open repair.

Informed consent for publication of operative images and data was obtained from the patient.

After insertion of a lumbar cerebrospinal fluid drain, the patient was positioned in a right lateral decubitus position. A thoracoabdominal incision was made extending from the fifth intercostal space to the umbilicus. The diaphragm was

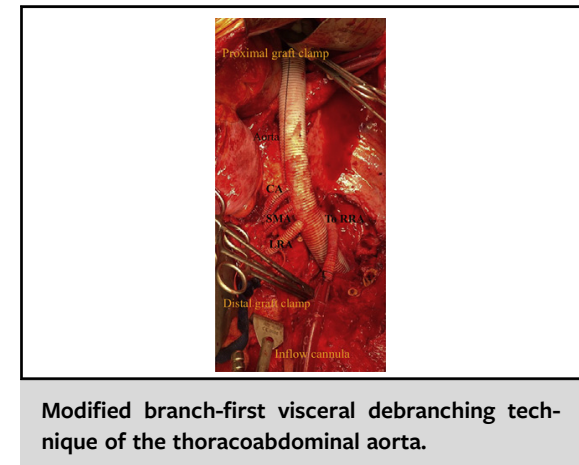

CENTRAL MESSAGE

We describe a modified branchfirst technique of open thoracoabdominal aortic aneurysm repair that helps minimize the overall morbidity and mortality associated with the procedure.

See Commentaries on pages 32 and 34 .

divided, and medial visceral rotation was carried out to expose the thoracoabdominal aorta. Short segments of the descending thoracic and infrarenal aorta were dissected and controlled. Subsequently, the left renal artery (LRA), celiac axis (CA), and superior mesenteric artery (SMA) were circumferentially dissected and controlled at their origin. After systemic heparinization, the descending thoracic aorta was cannulated and connected to a Y-circuit, with one limb perfusing a branched Coselli graft $(24 \times 8 \times 8 \times 7 \times 7 \mathrm{~mm}$; Terumo Cardiovascular Systems, Ann Arbor, Mich) through a side branch and the other limb clamped, ready to be connected to CPB if needed (Figure 1, A). The CA, SMA, and LRA were divided, and stumps were ligated and anastomosed to the graft sequentially in an endto-end fashion, with ischemia times of $10 \mathrm{~min}, 12 \mathrm{~min}$, and 14 min, respectively (Figure 2, A). After completion of each anastomosis, the branches were declamped to restore respective visceral perfusion (Figure 2, B). After 2 clamps were placed on the proximal aorta (Figure 1, B), the proximal aortic anastomosis was completed, and pulsatile antegrade flow was restored to the viscera (Figure 2,C). The 

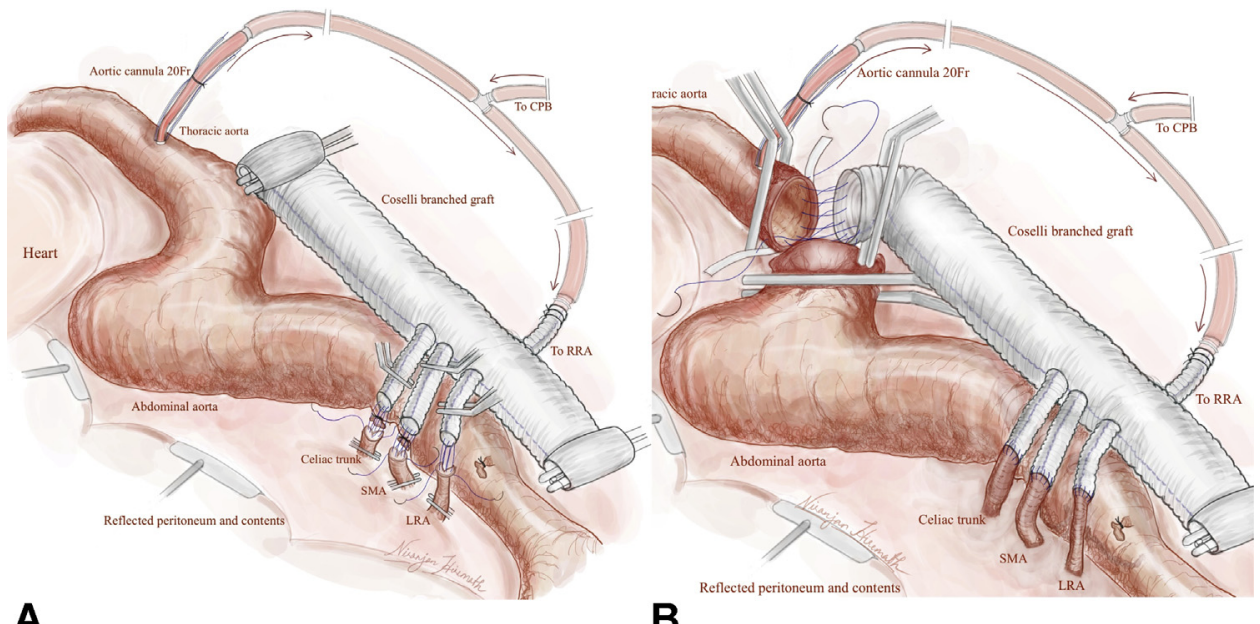

B

FIGURE 1. A, Illustration demonstrating the modified branch-first technique that depicts the retroperitoneal exposure of the thoracic aorta, abdominal aorta, and visceral branches. The right renal artery $(R R A)$ is not visible at this stage and is accessed later after opening the aneurysmal sac. The branched thoracoabdominal graft is placed in a $z$-axis orientation to the aorta with the ends clamped and rolled. The graft is perfused and pressurized via the graft (RRA) limb with inflow from the descending aortic cannula connected to a Y-circuit (arrows demonstrate the direction of blood flow within the closed circuit). The other clamped limb provides inflow to the graft from the cardiopulmonary bypass $(C P B)$ pump if the need for full CPB support arises. Graft anastomoses to the celiac artery (CA), superior mesenteric artery (SMA), and left renal artery (LRA) are performed in a sequential manner, and blood flow is restored on completion of each anastomosis. B, The proximal aorta is clamped just below the arterial cannula, 2 to 3 inches below the proximal aneurysmal aorta; the aortic stump is transected; and the proximal anastomosis is completed. Note that there is continuous visceral perfusion via the graft inflow circuit while the proximal aortic anastomosis is performed. The aneurysmal sac is still intact at this stage. (Original illustration by First author.)
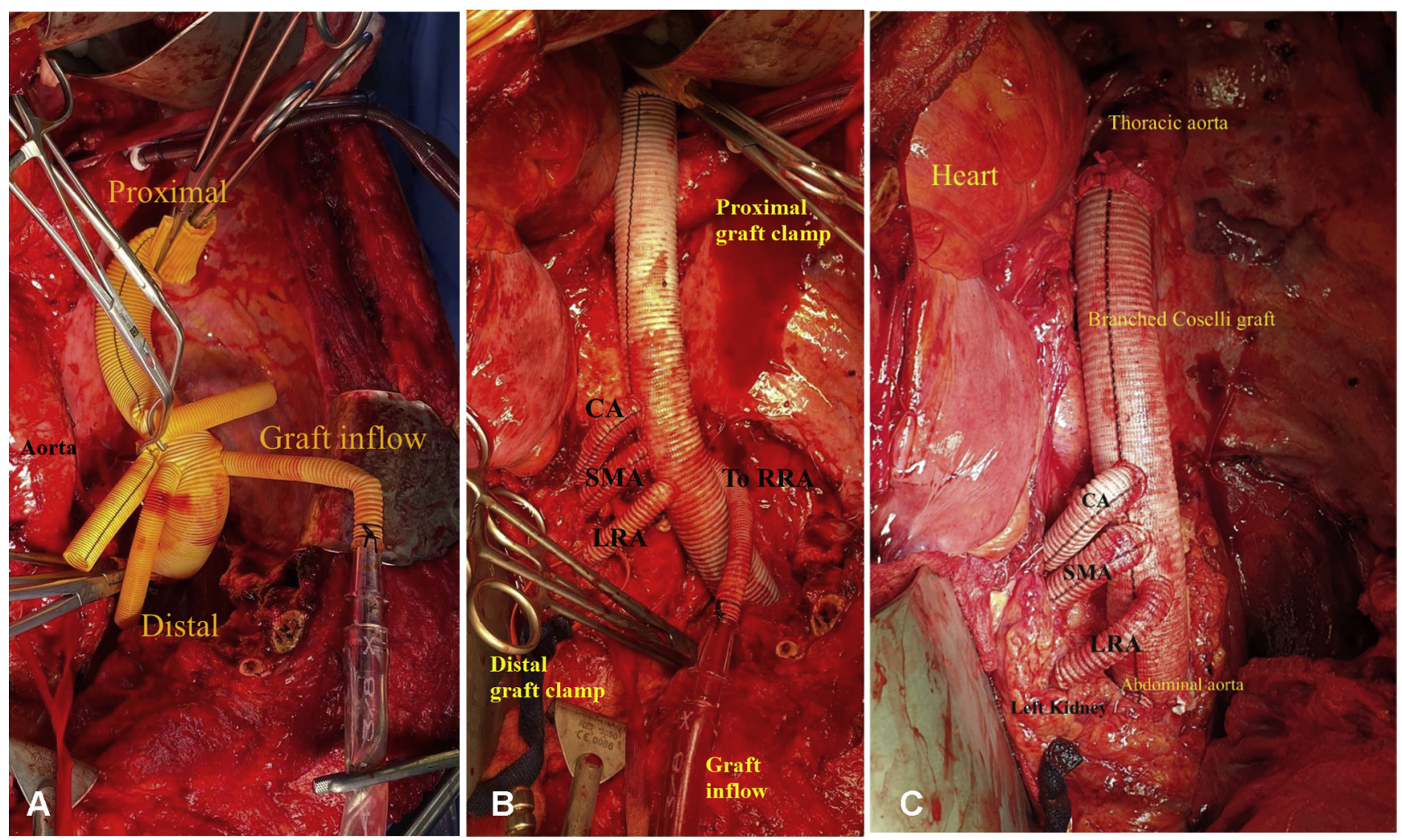

FIGURE 2. A, Thoracoabdominal branched graft placed adjacent to the aorta with the graft inflow cannula secured. B, Visceral debranching completed while the proximal and distal graft ends remain clamped and the graft is perfused through the closed arterial circuit. C, Final lie of the aortic graft after completion of all anastomoses. $C A$, Celiac artery; $S M A$, superior mesenteric artery; $R R A$, right renal artery; $L R A$, left renal artery. 


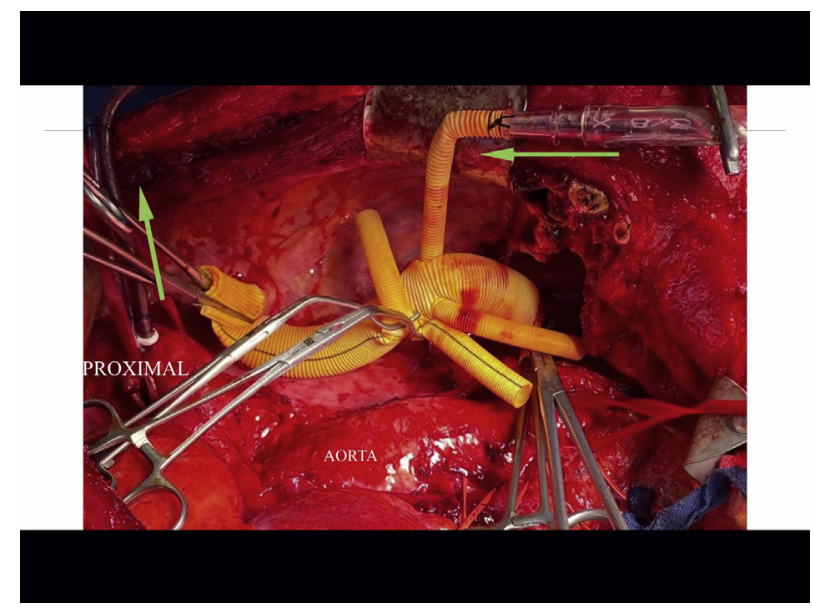

VIDEO 1. Short video presentation explaining the principles and features of open repair of extent-III thoracoabdominal aortic aneurysm using the modified branch-first technique. Video available at: https://www.jtcvs. org/article/S2666-2507(21)00248-0/fulltext.

infrarenal aorta was clamped, and the aneurysm sac was opened to expose the right renal artery orifice. The right kidney was cooled with $300 \mathrm{~mL}$ of cold Ringer's lactate. The inflow cannula was then disconnected from the graft limb and anastomosed to the right renal artery (RRA). The total ischemia time of the RRA was 30 minutes. Finally, the distal infrarenal aortic anastomosis was completed, the intercostal and lumbar vessels were oversewn, and distal body circulation was restored. The patient maintained adequate urine output throughout the procedure, and no banked blood was transfused (Video 1).

The patient was discharged on postoperative day 10 after being treated for left lung atelectasis and spinal headache. On follow-up, CT aortography showed satisfactory repair of the aorta with adequately perfused visceral branches. The patient returned to his active lifestyle at 6 weeks after surgery.

\section{DISCUSSION}

TAAA surgeries are notoriously time-consuming and morbid, are associated with visceral and spinal cord ischemia, and necessitate high amounts of blood product consumption. Numerous strategies have been described over the last 50 years, including deep hypothermic circulatory arrest, ${ }^{1}$ left heart bypass or full $\mathrm{CPB},{ }^{2}$ special spinal protection techniques, and hybrid surgical techniques. ${ }^{3}$ The branch-first technique for TAAA repair was originally described by Matalanis and Ch'ng, ${ }^{4}$ where the visceral debranching is performed first with a trifurcation graft, which is perfused by attaching an aortic cannula to one of its branches, and finally implanting onto the aortic graft. In principle, this technique provides uninterrupted visceral blood flow during the debranching phase until it is time to perform the graft-to-aorta anastomosis, when perfusion to viscera ceases. Consequently, it reduces spinal cord ischemia time, decreases aortic cross-clamp time, and eliminates the need for a separate visceral perfusion circuit from the $\mathrm{CPB}$ machine or the need for deep hypothermic circulatory arrest. In our modification, we used a Coselli graft, with inflow to the RRA graft limb from the descending aortic cannula. This allowed performance of the proximal aortic anastomosis while still perfusing the visceral limbs, thereby decreasing visceral ischemia time. The modified branchfirst technique has advantages over a single-origin visceral perfusing limb, which has lower inflow and is more prone to kinking and thrombosis, jeopardizing visceral blood flow. ${ }^{4}$ Alternatively, the graft inflow can be derived from the axillary artery or via a partial left heart bypass connected directly to the graft limb.

\section{CONCLUSIONS}

The modified branch-first technique is an effective and safe strategy that can help minimize the overall morbidity and mortality associated with open TAAA repair. Adequate preoperative planning is imperative for the hassle-free performance of this extensive procedure.

\section{References}

1. Kouchoukos NT. Thoracoabdominal aortic aneurysm repair using hypothermic cardiopulmonary bypass and circulatory arrest. Ann Cardiothorac Surg. 2012;1: 409-11.

2. Coselli JS, Bozinovski J, LeMaire SA. Open surgical repair of 2286 thoracoabdominal aortic aneurysms. Ann Thorac Surg. 2007;83:S862-4; discussion S890-2.

3. Orozco-Sevilla V, Weldon SA, Coselli JS. Hybrid thoracoabdominal aortic aneurysm repair: is the future here? J Vis Surg. 2018;4:61.

4. Matalanis G, Ch'ng SL. Thoracoabdominal aortic aneurysm-the branch first technique. Semin Thorac Cardiovasc Surg. 2019;31:708-12. 